\title{
Regulation of Hematopoietic Activity Involving New Interacting Partners (RRAGC \& PSMC2, CKAP4 \& MANF and CTR9 \& CNTNAP2)
}

\author{
Swati Sharma ${ }^{1}$, Gurudutta U. Gangenahalli2*, Upma Singh ${ }^{3}$ \\ ${ }^{1}$ Department of Pharmacology, All India Institute of Medical Science (AIIMS), New Delhi, India \\ ${ }^{2}$ Stem Cell \& Gene Therapy Research Group, Institute of Nuclear Medicine \& Allied Sciences, New Delhi, India \\ ${ }^{3}$ Department of Applied Chemistry, School of Vocational Studies \& Applied Sciences, Gautam Buddha University, Greater Noida, \\ India \\ Email: *gangenahalligurudutta@gmail.com
}

How to cite this paper: Sharma, S., Gangenahalli, G.U. and Singh, U. (2020) Regulation of Hematopoietic Activity Involving New Interacting Partners (RRAGC \& PSMC2, CKAP4 \& MANF and CTR9 \& CNTNAP2). CellBio, 9, 123-141.

https://doi.org/10.4236/cellbio.2020.93007

Received: August 13, 2020

Accepted: September 27, 2020

Published: September 30, 2020

Copyright $\odot 2020$ by author(s) and Scientific Research Publishing Inc. This work is licensed under the Creative Commons Attribution International License (CC BY 4.0).

http://creativecommons.org/licenses/by/4.0/

\section{(c) (i) Open Access}

\begin{abstract}
Hematopoietic stem cells (HSCs) are tissue-specific cells giving rise to all mature blood cell types regulated by a diverse group of hematopoietic cytokines and growth factors that influences the survival \& proliferation of early progenitors and differentiation mechanisms by modulating the functional activities of HSCs. In this study, the functional yet distinctive role of three novel combinations of gene pairs RRAGC \& PSMC2; CKAP4 \& MANF; and CTR9 \& CNTNAP2 have been newly identified. These novel combinations of genes were confirmed and expressed in K562 human leukemic cell line in the presence of cytokine combination (IL-3, FLT-3 and SCF) using RT-PCR and siRNA-mediated gene knock down strategy. This study signifies the synergistic role of gene pairs in different molecular activities like ubiquitination or proteasomal degradation, calcium mobilization, dopamine signaling.
\end{abstract}

\section{Keywords}

Hematopoietic Stem Cells (HSCs), Signal Transduction, Haematopoiesis

\section{Introduction}

Hematopoietic stem cells (HSC) are adult stem cells that have the potential to self-renew and differentiate into specialized blood cells and play a key role in hematopoiesis, maintaining homeostasis and immune responses. The programming of stem cells has been determined by a complex interplay between the in- 
trinsic genetic factors and their environment, including the cytokines and growth factors. Critical signals from bone marrow microenvironment are received by the HSCs for cell programming, where cytokines, stromal cells, and ECM molecules interact in niche to onset various intracellular signal transduction pathways. Transplantation of HSCs has shown several therapeutic applications in the treatment of blood diseases, inherited blood disorders, and autoimmune diseases [1] [2].

The cytokines exhibit pleiotropic actions either through an array of signaling components and transcription factors and can activate a variety of downstream signaling events or can produce overlapping or identical effects in the same target cell [3].

During the molecular pathway crosstalk, the binding of a ligand to its receptor initiates the specific protein-protein interactions and protein modifications in response to an external stimulus. In such events, the action of protein kinases, phosphatases \& phosphopeptide binding domains becomes a significant factor for translocation from upstream signaling molecule to downstream effectors to initiate developmental cascades [4]. Moreover, the common reaction motifs become linking points to classify interactions and provide a description of cascade pathways.

Interventions of stem cell research into clinical applications have been facilitated with chemical approaches by providing robust tools to precisely manipulate stem cell fate and function in-vitro to a sufficient number of safe, homogenous, and functional cells for cell therapy. However, several issues related to the complexity of the process \& long-term maintenance of transplanted cells and cost possess challenges to its success. Alternatively, another strategy of directly modulating endogenous tissue-specific stem/progenitor (or even more differentiated) cells in-vivo for therapeutic benefits may also be achievable to some extent.

In this study, the functional yet distinctive role of three novel combinations of interacting of gene pairs (RRAGC \& PSMC2, CKAP4 \& MANF and CTR9 \& CNTNAP2) have been elucidated in hematopoiesis, by identifying a newly interacting link between them (through identifiable enzymatic functions or specific subcellular localization). These pair of genes have been observed to be involved in different functional activities such as ubiquitination or proteasomal degradation, calcium mobilization, dopamine signaling etc., that are directly or indirectly linked to HSC proliferation. Further, these have been identified to be essential in HSC fates, and therefore, happening of these processes in HSCs by specific mechanism is not been addressed or shown earlier. Hence, on the basis of these novel potential interactions between the identified pair of genes, it is presumed that such functional activities in specific biological mechanisms must have been achieved through the new or an unknown molecule, in addition to their mechanism or interacting partners shown in other cells.

\section{Material \& Methods}

The distinctive role of three novel combinations of interacting of gene pairs 
(RRAGC \& PSMC2, CKAP4 \& MANF and CTR9 \& CNTNAP2) have been elucidated in the present study, by identifying a newly interacting link between them (through identifiable enzymatic functions or specific subcellular localization) through computational approaches (GeneMania software). Later, their expression through RT-PCR confirmed and established the findings of gene expression analysis of our earlier study [5] and thus validate the novel pair of genes with an unexplored interaction/role by showing their maximum time of expression in K562 human leukemic cell line in the presence of cytokine combination (IL-3, FLT-3 and SCF) through siRNA-mediated gene knock down strategy.

The K562, a human caucasian chronic myelogenous leukemia suspension cell line was obtained from National Centre for Cell Science (NCCS), Pune, India, and cultured in IMDM (Iscove's Modified Dulbecco's Medium) supplemented with 2 nM Glutamine and 10\% FBS (Fetal Bovine Serum) with regular medium change every 3 - 4 days. The culture conditions were maintained for $10^{4}$ to $10^{5}$ cells $/ \mathrm{mL}$ at $5 \% \mathrm{CO}_{2}$ and $37^{\circ} \mathrm{C}$. Upon attaining confluency of $80 \%-90 \%$, cells were plated for expansion, experiments or cryopreserved. Later, the plated cells were treated with cytokines combination (IL-3 $=10 \mu \mathrm{g}, \mathrm{FLT}-3=10 \mu \mathrm{g} \& \mathrm{SCF}=20$ $\mu \mathrm{g}$ ) in 6 sets (for 6 genes) and control separately. The plated cells were then incubated for 12 hours, 24 hours and 36 hours to perform the cell kinetic study. Then the gene expression (6 gene candidates) was confirmed through Q-RT-PCR technique. The Q-RT-PCR primers for 6 pair of gene of interest were designed using Primer3Plus software. The designed primer sequence specificity for each target gene was further confirmed by blast programme (http://blast.ncbi.nlm.nih.gov/). The primer sequence for each gene is given in Table 1:

Table 1. List of gene-specific Q-RT-PCR primers.

\begin{tabular}{cccc}
\hline s. No. & Gene & Primers $\left(5^{\prime}\right.$ to $\left.3^{\prime}\right)$ & $\mathbf{T}_{\mathrm{m}}\left({ }^{\circ} \mathrm{C}\right)$ \\
\hline 1 & Forward RRAGC & ACGGTGGTTGCATTAGTTCC & 59.9 \\
2 & Reverse RRAGC & AAGGGGACTGTGGGAAAACT & 50.0 \\
3 & Forward PSMC2 & AAGCAAGGAAGGTGCTGTGT & 59.9 \\
4 & Reverse PSMC2 & AAGGCAATATCCCCCTCATC & 60.1 \\
5 & Forward CKAP4 & TCTTCCTGACGCATCTTGTG & 60.0 \\
6 & Reverse CKAP4 & GTCGAAACCTGTGCATTCCT & 60.1 \\
7 & Forward MANF & CTGAGCACAGTGGACCTGAA & 60.0 \\
8 & Reverse MANF & GGTGCAACAGAGATTGAGCA & 60.0 \\
9 & Forward CTR9 & AGTCAGGGAGAAGCCACTCA & 59.9 \\
10 & Reverse CTR9 & TTGTTGGATCCCTCTGGTTC & 60.0 \\
11 & Forward CNTNAP2 & GGATGCTCTACAGCGACACA & 60.0 \\
12 & Reverse CNTNAP2 & TCTCCATTCCAATCCAGAGG & 60.0 \\
\hline
\end{tabular}


After confirmation of expression of the genes of interest, the gene knock down was carried out through siRNA transfection method to establish the functional role of genes of interest in molecular signaling.

The study the effect of siRNA-mediated gene knock-down, the viability of the cells was measured by using Trypan Blue dye (Figure 1) exclusion procedures for viable cell counting. This method is based on the principle that live (viable) cells do not take up certain dyes, whereas dead (non-viable) cells do. Staining facilitates the visualization of cell morphology.

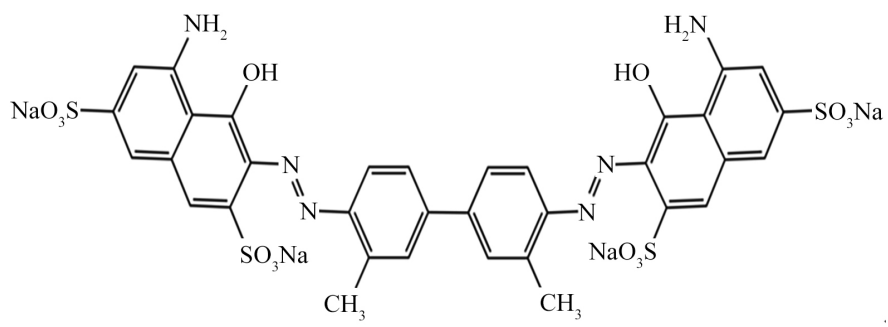

Figure 1. Trypan blue dye.

(3Z,3'Z)-3,3'-[(3,3'-dimethylbiphenyl-4,4'-diyl)di(1Z)hydrazin-2-yl-1-yli dene]bis(5-amino-4-oxo-3,4-dihydronaphthalene-2,7-disulfonic acid).

The cell viability/proliferation count was calculated by using the following formula:

$$
\text { Cell Viability }(\%)=\frac{\text { total viable cells }(\text { unstained })}{\text { total cells }(\text { stained } \& \text { unstained })} \times 100
$$

\section{Results}

\subsection{Interaction of the Genes RRAGC and PSMC2}

\subsubsection{RRAGC/Rag C-mTOR Signaling Dynamics}

In an environment, where nutrient availability is variable, organisms survive through evolved mechanisms for an efficient transition between anabolic and catabolic states. In case of mammals, for instance, this mechanism has been performed by protein kinase mTOR (mammalian/mechanistic Target of Rapamycin) anchored signaling network. The pathway responds to diverse environmental cues and has been simultaneously involved in different processes thereby either generating or using large amounts of energy and nutrients. It has been noticed that mTOR signaling influences major cellular functions like growth and proliferation. In many of the human diseases, including cancer, type 2 diabetes, obesity, and neuro-degeneration, mTOR deregulation has been observed, and hence, significant ongoing pharmacological efforts have been made to target this signaling pathway. mTOR has been found to be a target of rapamycin/sirolimus, a macrolide produced by the bacteria $S$. hygroscopicus, which gained attention because of its antiproliferative properties. mTOR, a serine/threonine protein kinase has shown to be a downstream effector of the PI3K/AKT pathway, that forms two multiprotein complexes-mTOR complex 1 (mTORC1) and 2 (mTORC2) [6]. 
mTORC1 pathways (sensitive to rapamycin) have been commonly activated during oncogenesis through Ras-ERK and PI3K-mTORC1 signaling pathways (thereby activating S6K1 and 4EBP1), as a key regulator of cell survival, proliferation, metabolism, and motility [7] as shown in Figure 2. However, mTORC2 has been found resistant to rapamycin and is generally insensitive to nutrients and energy signals, activates PKC- $\alpha$ and AKT and regulates the actin cytoskeleton $[8]$.

RRAGC/Rag C is a Rag A-interacting protein, which belongs to Ras sub-family of Ras-like small G proteins family that acts as a key regulator of cell growth and is essentially required for signal transduction pathways, initiated by receptor tyrosine kinases. Ras-like small G proteins (like Ras, Rab, Rho, Ran \& ARF) are a monomeric unit, which binds to the guanine nucleotides, GTP or GDP, and function as molecular switches. It also plays a significant role in cell growth, differentiation, and protein traffic between those compartments within the cells having Rag D [9]. The binary switch of Ras proteins family has shown to be regulated by many Ras guanine nucleotide exchange factors (RasGEFs) namely Son-of-sevenless (SoS), Ras guanine nucleotide release-inducing factors (RasGRFs) and Ras guanine nucleotide releasing proteins (RasGRPs) [10].

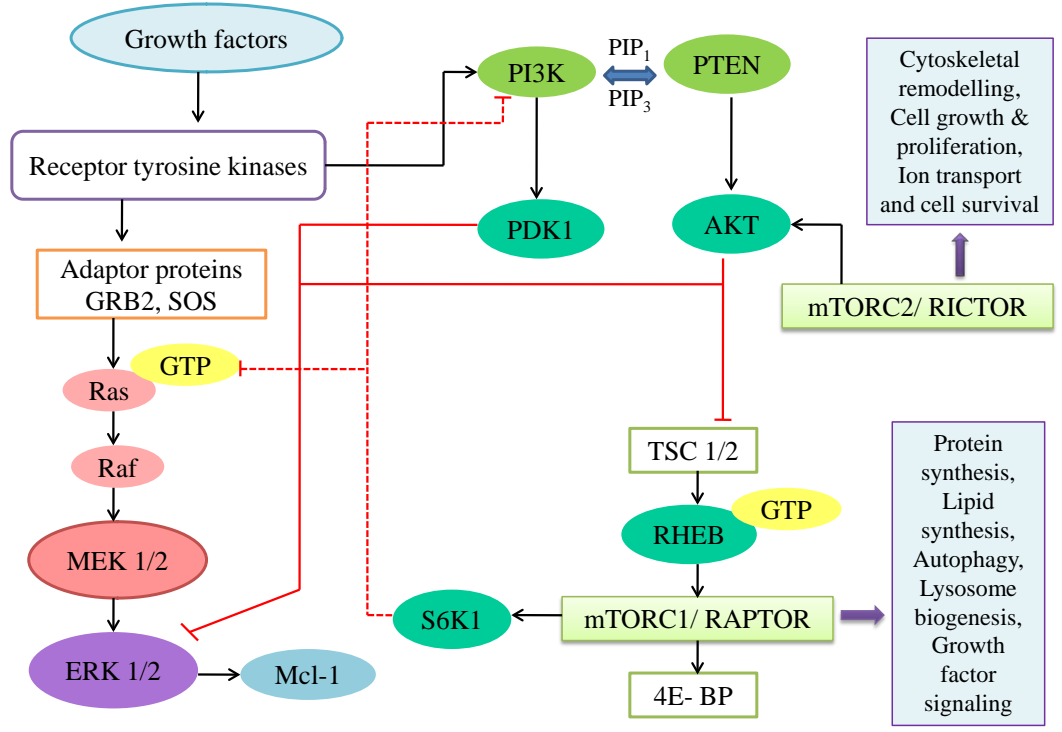

Figure 2. The Ras-MAPK and PI3K-mTOR pathways. Receptor tyrosine kinases transduces the signal factors and growth factors via adaptor proteins (such as GRB2 and the guanine-nucleotide exchange factor SOS), thereby activating Ras and other downstream cascade targets, like Raf and MEK1/2, resulting in the activation of ERK1/2 and upregulation of myeloid cell leukemia sequence 1 (Mcl-1). The signaling also proceeds through PI3K pathway, which activates pyruvate dehydrogenase kinase isozyme 1 (PDK1) and AKT, activating mTORC1/regulatory-associated protein of mTOR (RAPTOR) by relieving it of the inhibitory influence of tuberous sclerosis $1 / 2$ (TSC1/2) complex and ERK1/2, i.e. the inhibition of mTOR promotes cell death/Autophagy and Lysosome biogenesis. The mTORC2/RICTOR, on the other hand also stimulates the AKT pathway and is responsible for cytoskeleton remodeling and cell growth \& proliferation. 
Particularly, Rag C has GTP-binding motifs in its N-terminal region and has been shown to bind significantly to [3H]GTP and [3H]GDP in-vitro. Recent findings have shown that Rag A and Rag $\mathrm{C}$ share a mutual binding region in their C-terminal regions downstream of the GTP binding region, and it was proposed that Rag C and Rag D belong to the Rag A subfamily of the Ras-like small $G$ protein superfamily. Rag A has also been shown to be co-localized with Rag C and Rag D in BHK21 cells, which supported the finding that Rag A may form a hetero-dimer with Rag C or with Rag D. However, the C-terminal domains of Rag C/Rag D are similar to those of Rag A, which have found to be larger than other known members of mammalian Ras family and certainly not related to guanine nucleotide binding. A potential leucine zipper motif, implicated in dimer formation has been found just downstream of the nucleotide-binding or the effector region in the C-terminal region of Rag A/Rag C [9].

By the high sequence similarity and findings that mutation of Rag A suppressed the prp20/mtr 1 mutation of $S$. cerevisiae, it has been shown that Rag A is a human homologue of GTR1. It has been reported that Gtr1p is located within both the cytoplasm and the nucleus and play an important role in cell growth. Since Rag C and Rag D belong to Rag A/Gtrlp subfamily, but having different G2 and G3 motifs than that of other G proteins has been highly conserved between Rag A/Gtr1p and Rag C/Gtr2p [9] [10].

Depending on the nucleotide-bound forms, T21L (GDP) and Q66L (GTP), Rag A has been shown to change its subcellular localization and probably shuttles between the nucleus and the cytoplasm. It also appears very likely that Rag A/Gtr1p may influence Rag C and Rag D/Gtr2p regarding their subcellular localization. The concentration of GTP has been about 30 times higher in the nucleus than that of GDP concentration, which may result in the preferential production of the GTP form of Rag A within the nucleus. It may be possible that through heterodimer formation, the GTP form of Rag A/Gtr1p is responsible for binding and transporting the Rag C/Gtr2p from nucleus to the cytoplasm [9].

During the phase of insufficiency of nutrients/growth factors suppresses mTOR kinase (and thus Rag C/Rag D heterodimer) rapidly which stimulates overall protein degradation by autophagy. This results in the arrangement of essential amino acids for new protein synthesis and energy production. It has been demonstrated that inhibition of mTOR signaling increases overall protein breakdown by the ubiquitin proteasome system by accelerating the ubiquitination of cellular proteins, for example, HMG-CoA synthase, which is critical for cholesterol biosynthesis [11].

\subsubsection{Role of PSMC2 in Ubiquitination of Proteins}

$26 \mathrm{~S}$ protease regulatory subunit 7 , commonly known as $26 \mathrm{~S}$ proteasome AAAATPase subunit Rpt1, is an enzyme that is encoded by the PSMC2 gene in humans, is one of the 19 essential subunits of a $19 \mathrm{~S}$ proteasome complex. It has been shown that six subunits of $26 \mathrm{~S}$ proteasome AAA-ATPase/Rpt1 together with other units Rpt2, Rpt3, Rpt4, Rpt5, Rpt6 and four non-ATPase subunits 
(Rpn1, Rpn2, Rpn10, and Rpn13) constitutes the base subcomplex of 19S regulatory particle for proteasome complex as per the KEGG pathway [12] as shown in Figure 3.

It has been evident that $26 \mathrm{~S}$ proteasome subcomponent (a multiprotein complex) is involved in the ATP-dependent degradation of ubiquitinated proteins. The multiprotein complex has shown to be crucial for the maintenance of protein homeostasis by removing misfolded/damaged proteins, those impairing the cellular functions. Hence, the proteasome contributes to cellular processes such as cell cycle progression, apoptosis, or DNA damage repair. PSMC2, a member of a hetero-hexameric ring of AAA proteins unfolds ubiquitinated protein targets that have been concurrently translocated into a proteolytic chamber and degraded into peptides [12].

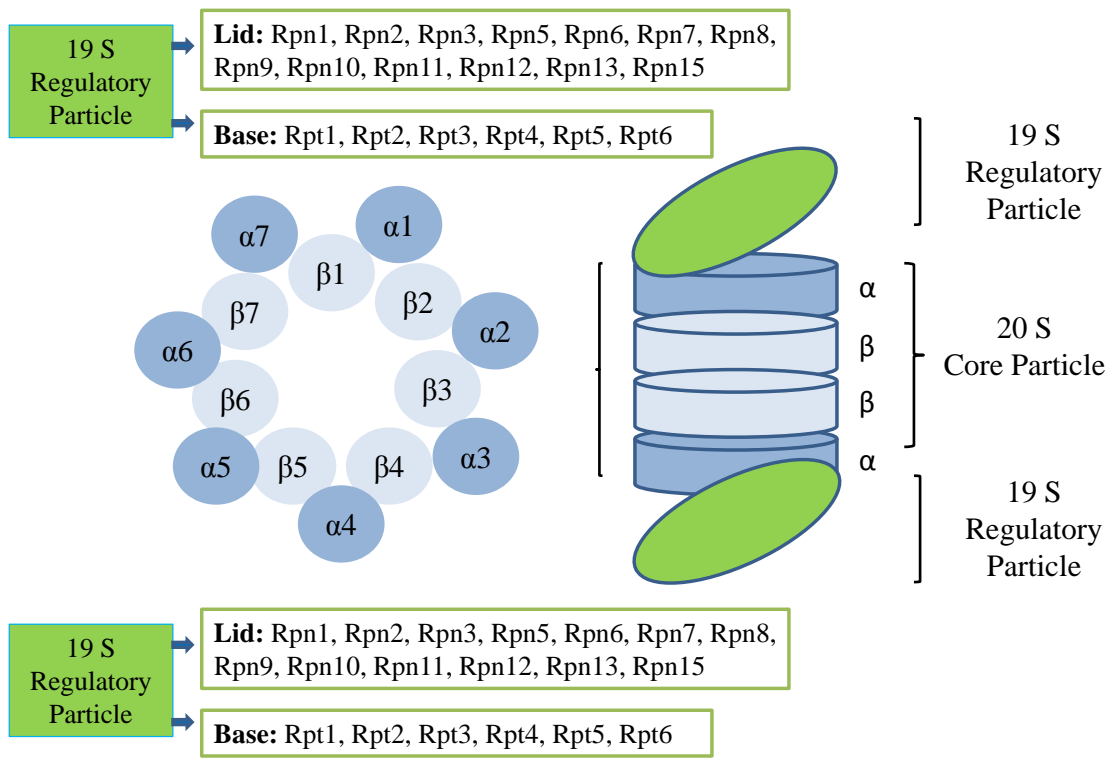

Figure 3. Structure of Proteasome. The $26 \mathrm{~S}$ proteasome is comprised of cylindrical $20 \mathrm{~S}$ Core unit (proteolytic) and two 19 S Regulatory units. The regulatory 19S unit recognises and binds with ubiquitinated proteins, catalyses their deubiquitination and unfolding to prepare them for proteolysis which occurs inside the $20 \mathrm{~S}$ unit. The $20 \mathrm{~S}$ core proteolytic unit consists of 2 pairs of 14 hetero polypeptides ( $\alpha$ and $\beta$ ) arranged in 4 stacked rings.

\subsubsection{Pathway Cross-Activation by RRAGC \& PSMC2}

An autophagy-lysosome system and the ubiquitin proteasome system (UPS) constitutes a major tool for degradation of proteins in eukaryotic cells. Cytoplasmic proteins are transported to autophagic vacuoles for degradation through the process of macroautophagy, where autophagosome has been formed upon starvation or mTOR inhibition. Further, the degradation of proteins either short-lived or misfolded or long-lived with bulk cellular components is done by highly selective UPS. This involves attachment of ubiquitin (Ub) chain to the substrate through the sequential actions of a Ub-activating enzyme (E1), Ubconjugating enzymes (E2s), and one of the cell's many Ub ligases (E3s) [11]. Here, $26 \mathrm{~S}$ proteasome (encoded by the PSMC2 gene) has been shown to rapidly 
degrade the proteins (short-lived proteins) conjugated with Ub chains using proteasome-associated deubiquitinating enzymes (DUBs) and show up linkage with Rag C/RRAGC as shown in Figure 4.

The three significant anabolic roles of mTORC1 namely, ubiquitination (through UPS), lysosomal pathway and autophagy activation have been simultaneously linked to each other and involve distinct downstream mechanisms, since mTORCl's suppression of the UPS has shown to be independent of its targets, thereby controlling autophagy or translation [11]. The immediate activation of the UPS can synergize with the stimulation of autophagy to eliminate toxic proteins. However, emphasis needs to be laid on the elucidation of the global mechanism governing the proteasomal degradation through these modes of action, which would provide a better understanding of the synergistic effect of RRAGC \& PSMC2.

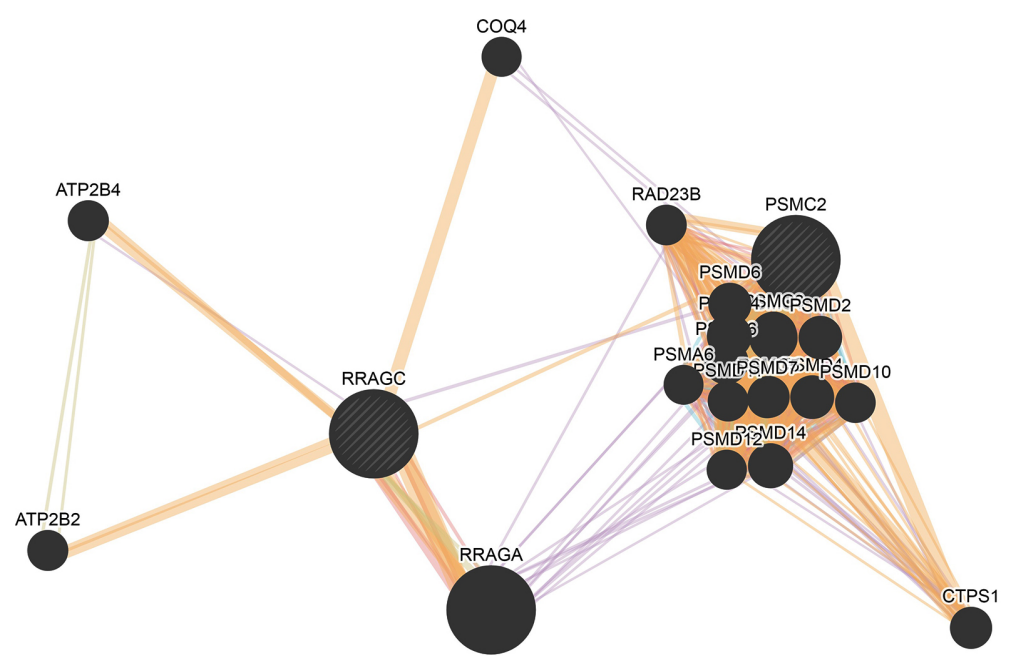

Figure 4. Protein interaction network showing linkage of RRAGC \& PSMC2.

\subsection{Interaction of the Genes CKAP4 and MANF}

\subsubsection{Functioning of CKAP4}

Cytoskeleton-associated protein 4 (CKAP4; also known as p63, CLIMP-63, or ERGIC-63), a resident of the endoplasmic reticulum (ER)/Golgi intermediate compartment (ERGIC) is a $63 \mathrm{kDa}$, reversibly palmitoylated and phosphorylated belongs to the family of type-II transmembrane (TM) protein. Accumulating evidences have shown the existence of CKAP4 in multiple cellular compartments (i.e. ER, plasma membrane, and the nucleus) and its diverse roles in the regulation of several physiological and pathological processes, like interstitial cystitis, drug-induced cytotoxicity, pericullar proteolytic activity, and lung lipid homeostasis [13]. However, its major function has been to anchor rough ER to microtubules, organizing the overall structure of ER with respect to the microtubule network, on its localization to the ER. The rough ER provides the site for transmembrane/secretory proteins (made on polysomes) gets attached to ER membranes. The attachment of single unit i.e. an individual ribosome to the ER 
membrane has been mediated by a complex molecular apparatus having Sec61p at its core, which stimulated the signal sequence-mediated targeting, co-translational translocation, and processing of nascent polypeptide chains (the complex is referred as translocon complex-TC) [14].

It has been shown that CKAP4/CLIMP-63 interacts with microtubules via its cytosolic domain and probably MAP2. Moreover, the lumenal domain of CLIMP-63 (CKAP4) has shown to promote oligomerization and its complete immobilization in the plane of the ER membrane results in the formation of a network at the luminal face of ER membrane [14]. Further, in COS-1 cells, the over expression of certain CLIMP-63 mutants indicated the rearrangements and co-alignments of the ER membranes with microtubules [15].

As mentioned, microtubules play a significant role in stabilizing the ER structure through interaction with several microtubule binding proteins including CLIMP-63/CKAP4. The microtubule-binding domain of CKAP4 is unique as it has been found to only affected by high salt concentrations (>200 mM NaCl) and contain no Src homology 2 domain. The interaction of CLIMP-63/CKAP4 with microtubule and ER has an association site (in close proximity with ER stress proteins GRP78/94) which may also be related with ATF6 signaling mediating ER stress response induction [16].

Simultaneously, it has been proposed that organellar membrane proteins, found in the endomembrane system can bind directly to the cytoskeleton such as CLIMP-63 \& CLIMP (Golgi protein giantin). It has also been shown that only a small proportion of cells possess actively moving ER, while in most cells the ER has been found to be less dynamic (shown in living epithelial cells) [17]. Further, the findings suggested that the movements limited to a particular phase of the cell cycle that requires ER proliferation. However, CLIMPs may also regulate the dynamic interaction of organelles with the cytoskeleton in conjunction with CLIPs and molecular motors. Several lines of evidence have steadily confirmed the role of CLIMP-63/CKAP4 in de novo ER network formation through anchoring of tubulovesicular membranes to microtubules and thereby aiding motor protein-driven tubulation. However, the notion that some additional proteins have been required for the regulation of the same interactions in-vivo, specifically, during mitosis when the microtubules are required for spindle formation has also been supported by research.

Besides, CKAP4 has been evident to involve in an extensive number of functions such as folding, processing assembling, sorting, synthesizing, degrading, translocating, and glycosylating secretory and membrane proteins; synthesizing and metabolizing lipids; regulating intracellular calcium. Further, this luminal protein (CKAP4) has also been found to interact with a KDEL, an ER retention motif in the ER-Golgi intermediate compartment [18].

\subsubsection{Mechanism of MANF}

Mesencephalic astrocyte-derived neurotrophic factor (MANF) is an $18-\mathrm{kDa}$ soluble protein which has also shown to be localized to the luminal endoplasmic 
reticulum (ER). Its stress has shown to stimulate MANF expression and secretion. MANF has been found to regulate dopaminergic neuron development in Drosophila and zebrafish [19]. While, neuroprotective effects of MANF has been confirmed in animal models of neurodegeneration, without an understanding of their underlying mechanism [20].

MANF has found to be expressed in several tissue and cell types basally and also an increase in its level has been observed in response to accumulation of misfolded proteins in the SR/ER. Recent studies have shown that extracellular MANF protects against cell death in response to a variety of stresses, including oxygen and nutrient deprivation or ischemia [19].

In support of the findings that extracellular MANF protects the cultured cardiac myocytes, the mechanism of MANF secretion from ventricular myocytes was examined. Proteins like atrial natriuretic factor (ANF) have been secreted by Neonatal rat ventricular myocytes at rates dictated by its expression levels [21]. Hence, it has been hypothesized that MANF secretion would vary in coordination with its expression, as reported for other constitutively secreted proteins. The hypothesis has been tested by treating cultured cardiac myocytes for $20 \mathrm{~h}$ with tunicamycin (TM), thapsigargin (TG), and DTT, which resulted in increasing the expression of ER stress response genes thereby inhibiting the glycosylation, decreasing ER calcium and altering ER redox status, respectively. Each of these compounds has shown the increased expression of two ER stress well-known gene products-glucose-regulated proteins-GRP94 and GRP78. However, MANF was expressed at moderate levels, which increased in response to each ER stressor in the absence of ER stress.

\subsubsection{CKAP4 and MANF Interaction}

CKAP4 is associated with a number of functions such as folding, processing assembling, sorting, synthesizing, degrading, translocating, and glycosylating secretory and membrane proteins; synthesizing and metabolizing lipids; regulating calcium concentration, it has been likely to also interact with KDEL, an ER retention motif in the ER-Golgi intermediate compartment [18].

KDEL, an ER stress receptor along with GRP78 has also been reported to regulate MANF retention in the SR/ER of cardiac myocytes and HeLa cells. It has also shown that cells are protected by MANF after its secretion without a clear understanding of the conditions of its secretion [22]. However, in most of the conditions, it has been noted that MANF was not found secreted but retained in cells. Moreover, secretion of MANF has also been found to depend upon the depletion of calcium which reduces its strength of retention, involving its close interaction with the ER resident chaperone, GRP78. The threshold concentration of calcium required to dissociate the MANF-GRP78 complex fell within the range of ER calcium concentrations in cells treated with agonists that mobilize ER calcium in neurons [23] and cardiac myocytes in case of hypoxia [24], and in the ischemic brain and heart [25]. Thus, over such physiological concentration range (in response to ER calcium depletion), there has been a possibility that 
extracellular MANF could function as autocrine or paracrine to protect cells from death [26].

Also, the interaction of CLIMP-63/CKAP4 with microtubule and ER has an association site (in close proximity with ER stress proteins GRP78/94) may be related with ATF6 signaling which mediates ER stress response induction [16]. It has been apparent that GRP78 also binds to ATF6, an ER transmembrane protein, by conditionally retaining it in the ER. Consistently, it also has an ability to retain MANF in the ER, analogously to its ability to retain ATF6 in the ER, suggesting the signaling role of MANF during ER stress, but in only under a state of depleting calcium, indicating its specific role in ER calcium dysregulation [26]. However, a linkage needs to be further established on the synergistic role of CKAP4 and MANF in ER/Golgi signaling by either binding to KDEL or through ATF6 in close proximity with GRP78/GRP94, which has been shown in the present study using computational tools (as shown in Figure 5).

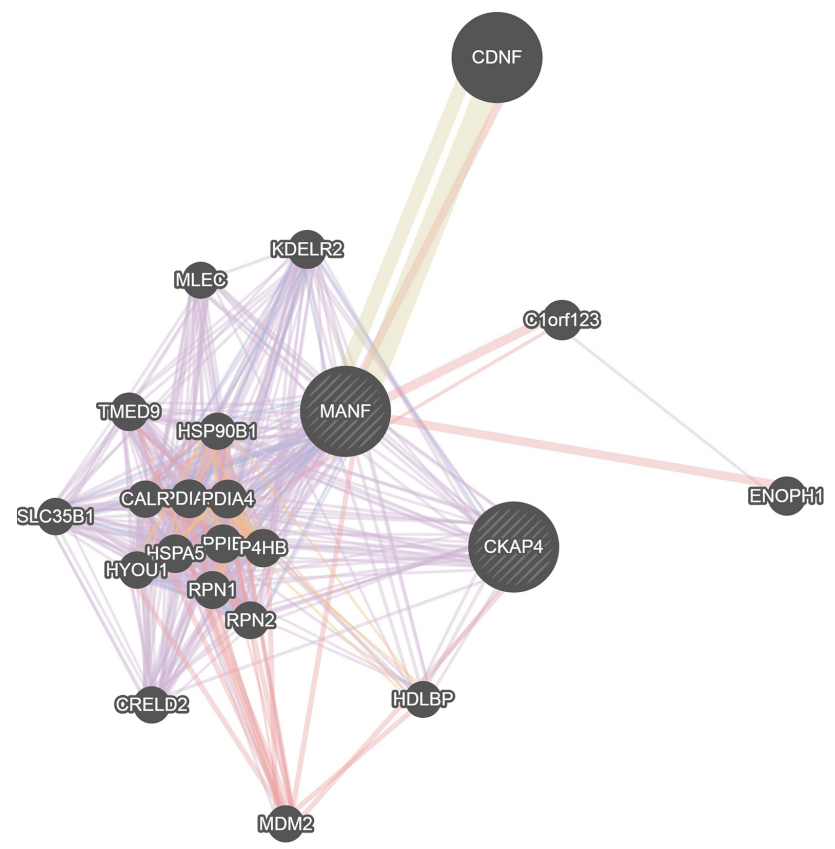

Figure 5. Protein interaction network showing linkage of CKAP4 \& MANF.

\subsection{Interaction of the Genes CTR9 and CNTNAP2}

\subsubsection{Significance of CTR9}

CTR9 is a key unit of human RNA polymerase II (RNAPII)-associated factor complex (hPAFc), which possesses multiple functions during transcription by RNA polymerase II and regulates the development and maintenance of embryonic stem cell pluripotency [27]. CTR9 has shown to be involved in regulating the $\mathrm{ER} \alpha$-dependent transcription, i.e. it controls $\mathrm{ER} \alpha$ stability along with promoting the elongation of transcriptional activity. It has also been suggested to regulate ER $\alpha$ extranuclear kinase signaling pathway through MAPK and AKT, which plays a significant role in the proliferation of $\mathrm{ER} \alpha^{+}$breast cancer cells and tamoxifen resistance [28]. 
Also, a new insight of CTR9 has been provided by its characterization using a yeast two-hybrid system. CTR9 has been identified as a novel DAT binding partner and found to be expressed in dopaminergic neurons. It has been shown to form a stable complex with DAT in-vivo via GST pulldown and co-immunoprecipitation assays. The role of the SH2 domain of CTR9 in nuclear localization has also been demonstrated, which confirms that CTR9 localization is not restricted to the nucleus, as its function has been primarily illustrated in the transcription complex Paf1. Thus, the recent research indicates that CTR9 modulates DAT function by regulating its trafficking [29].

The dopamine (DA)3-mediated neurotransmission has been an important activity as it is directly associated with neurological and psychiatric disorders, like Parkinson disease, dystonia, schizophrenia, attention-deficit/hyperactivity disorder, Tourette syndrome, and drug addiction [30]. All of these neurological conditions share a common pathological mechanism, i.e. dysfunction of DA transmission. In this mechanism, DA transporter (DAT) plays a pivotal role in removing DA from the synaptic cleft via an ionic gradient-dependent reuptake mechanism [31]. DA has been drained from the extracellular space in much less than milliseconds, and then, DAT regulates the availability of DA and activates pre- and post-synaptic DA receptors.

The formation of the DAT-CTR9 complex has been shown to be mediated by a direct protein-protein interaction between the membrane-proximal portion of the intracellular tail of DAT and a 500-bp region of CTR9 that contains the three complete tetracopeptide repeat domains (TPRDs) - TPRD-7, -8, and -9. It also revealed that an enhanced activity of DAT in the presence of CTR9 has been not likely due to changes in the intrinsic properties of DAT (such as the turnover rate or the recognition of DA by the DAT ligand binding domain) but rather it has been due to an increased number of transporters expressed at the plasma membrane [29] (Gois SD. et al., 2015).

The mechanisms underlying this regulatory activity have not been well understood. For example, activation of PKC leads to DAT internalization has been demonstrated, however, its removal from the cell surface is not prevented on mutation of its DAT phosphorylation sites [32]. Moreover, many DAT binding partners have been suggested to be involved in the modulation of DAT stability, localization, trafficking, and activity. In this regard, a study showed that DAT activity is influenced by a direct interaction between the C terminus of DAT (DAT-CT) and -synuclein [33]. DAT function has also been shown to be regulated by its interaction with the PDZ containing-domain PKC-interacting protein-1 (PICK1), the focal adhesion protein HIC-5, receptor for activated C-kinase1 (RACK1), the SNARE component syntaxin-1A, parkin, the DA receptors D2 and D3, synaptogyrin-3, the GTPase Rin and the G protein subunits.

Interestingly, these findings confirmed a relationship between transcription and neurotransmission through trafficking of CTR9 between the nuclear and plasma membrane compartments. However, the fact that whether Janus face-like 
activity of CTR9 in each compartment is fully independent or through a shuttle function between the nucleus and the cytoplasm remains an unsolved ambiguity and an attempt for future research [29].

\subsubsection{Role of CNTNAP2}

Contactin associated protein-like 2 (CNTNAP2)/CASPR2 is a member of the neurexin family that plays a significant role in the vertebrate nervous system as cell adhesion molecules and receptors. It has shown to play a role in the formation of functionally distinct domains, which have been critical for saltatory conduction of nerve impulses in myelinated nerve fibers and has also been involved in localization of potassium channels within differentiating axons. This protein, like other members of neurexin proteins family, contains epidermal growth factor repeats and laminin $\mathrm{G}$ domains. It has also found to have an F5/8 type $\mathrm{C}$ domain, discoidin/neuropilin- and fibrinogen-like domains, thrombospondin $\mathrm{N}$-terminal-like domains, and a putative PDZ binding site. This gene also has an implication in multiple neuro developmental disorders which includes tourette syndrome, schizophrenia, epilepsy, autism, ADHD and intellectual disability (CNTNAP2 Gene, NCBI, 2017).

It has been confirmed that prenatal exposure to nicotine strongly influences the cytoskeleton reorganization and increase the expression of cytoskeletal proteins [34]. This has been further supported by overrepresented PANTHER Pathways such as Dopamine receptor-mediated signaling (P05912) and Nicotine pharmacodynamics (P06587) pathways. The nicotine exposure has also shown to be linked with reduced methylation at CNTNAP2 (cg25949550) both in offspring exposed to prenatal smoking and in adults. Since mutations in CNTNAP2 have been associated with several neurological \& development disorders, it is possible that the prenatal smoking-associated methylation in CNTNAP2 and its persistency until at least adolescence might represent an additional mechanism contributing to impaired neurodevelopment in children exposed to in utero tobacco smoke [35].

Consistently, it has also been shown that EPB41L1 and its related protein EPB41L3 interact with CNTNAP2/CASPR2 in the brain where they may anchor this axonal transmembrane protein to the actin cytoskeleton. It contains a potential C-terminal binding site for a PDZ domain, which is probably involved in its interaction with $\mathrm{K}^{+}$Channels [36].

\subsubsection{CTR9-CNTNAP2 Interaction}

CTR9, a unit of multiprotein complex hPAFc plays a regulatory role during transcription and regulates the development and maintenance of embryonic stem cell pluripotency. Additionally, it has been identified as a novel DAT binding partner, which modulates DAT function and regulates its trafficking. This regulatory mechanism has been poorly understood. But, some studies provide evidence of this signaling in bits and pieces. For example, as mentioned, activation of PKC leads to DAT internalization, but when all DAT phosphorylation 
sites have been found to be mutated, and its removal from the cell surface is not prevented. Several DAT binding partners have been suggested to be involved in the modulation of DAT stability, localization, trafficking, and/or activity. Another study showed that DAT activity has been affected by a direct interaction between the $\mathrm{C}$ terminus of DAT (DAT-CT) and -synuclein. DAT function has also shown to be regulated by its interaction with the PDZ containing-domain PKC-interacting protein-1 (PICK1), the focal adhesion protein HIC-5, RACK1, the SNARE component syntaxin-1A, parkin, the DA receptors D2 and D3, synaptogyrin-3, the GTPase Rin and the G protein subunits.

CNTNAP2/CASPR2, on the other hand, has also shown implications for neuro-developmental disorders. Its linkage with paranodin shows its association with 4.1B (one of the four paralogues of vertebrates-4.1R, $4.1 \mathrm{~N}, 4.1 \mathrm{G}$ and $4.1 \mathrm{~B}$ ). It is also shown that $\mathrm{t} 4.1 \mathrm{~B}$ has been associated with Caspr/paranodin at paranodes, and with Caspr2 at juxtaparanodes and 4.1R has been known to bind to the transmembrane protein glycophorin C in erythrocytes [37] [38] at GNP motif level, conserved in paranodin. The timing of the clustering of these proteins suggests that the targeting of transmembrane protein occurs first, which is likely triggered by interactions with glial proteins [39]. Interestingly, CASPR2 also contains a potential C-terminal binding site for a PDZ domain, probably involved in its interaction with $\mathrm{K}^{+}$channels. In contrast, Caspr/paranodin does not possess a canonical PDZ binding site but has a potential binding site for SH3 domains [40].

The findings indicate a possible interaction between CTR9 and CNTNAP2 as shown in Figure 6. Further, it has been proposed that the CTR9-CNTNAP2 interaction to be involved in dopamine-mediated signaling through transmission of ion via ionic gradient-dependent mechanism and potential C-terminal binding site for a PDZ domain.

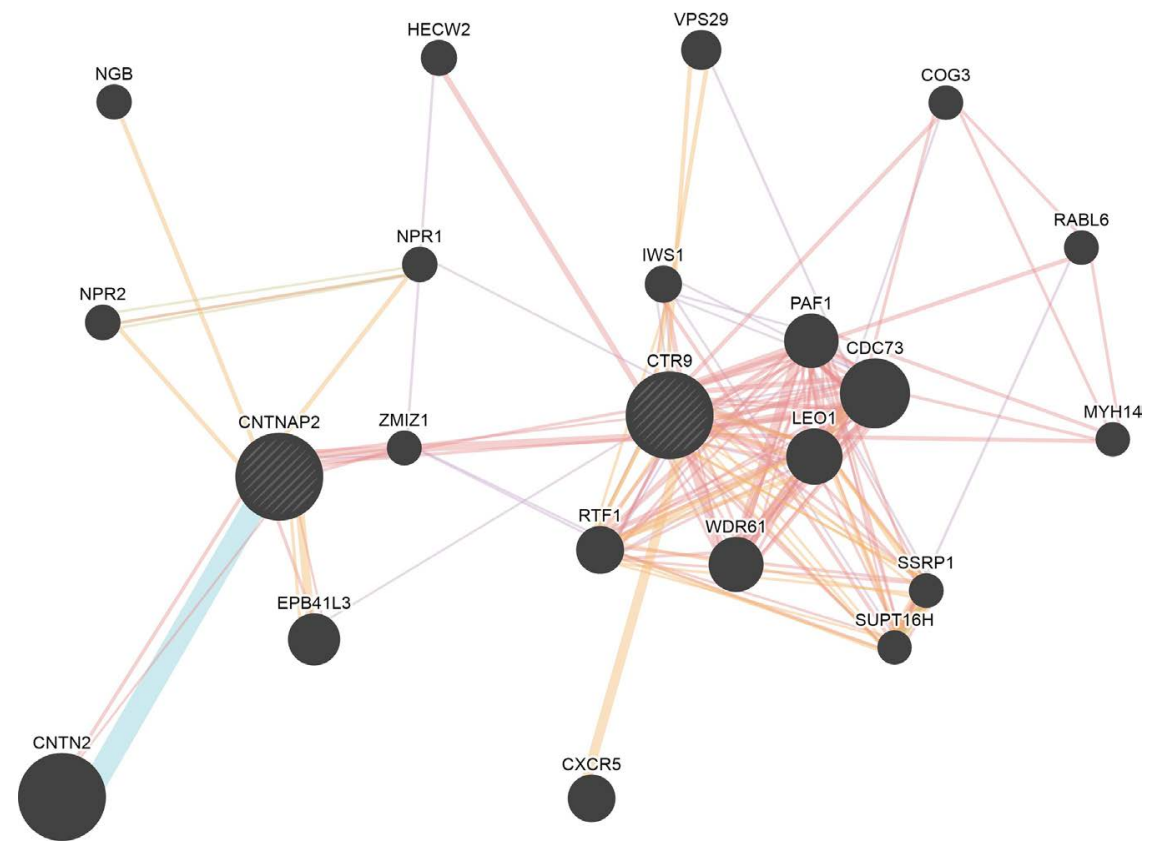

Figure 6. Protein interaction network showing linkage of CTR9 \& CNTNAP2. 
The findings of this research work (as discussed above) indicated the possibility of interaction of the novel pair of genes which was then validated through experimentation. Therefore, role of each of these unexplored gene candidates (RRAGC, PSMC2, CKAP4, MANF, CTR9 and CNTNAP2) was confirmed by showing their maximum time of expression (in different timescale) in K562 human leukemic cell line in the presence of cytokine combination, i.e. IL-3, FLT-3 \& SCF using Quantitative-Real Time Polymerase Chain Reaction (Q-RT-PCR) technique. Our research work indicated that RRAGC, CKAP4, MANF and CTR9 showed their maximum expression in K562 cell line at 12 hours, while PSMC2 showed maximum expression at 36 hours and CNTNAP2 did not showed expression. Since, CNTNAP2 expression was not observed, the pair CTR9 and CNTNAP2 was not taken for siRNA gene knock down procedures. Further, the siRNA-mediated gene knock down activity of 4 genes viz. RRAGC, PSMC2, CKAP4 and MANF were observed to confirm their role in proliferation and thus, established their functional significance.

Later, two pair of genes (RRAGC \& PSMC2 and CKAP4 \& MANF) were cultured again in K562 cell line, transfected, and optimized using their gene specific siRNAs. The gene knock down activity was observed in all of these cases separately and then the viability of cells (or proliferation count) was measured by using Trypan Blue dye

((3Z,3'Z)-3,3'-(3,3'-dimethylbiphenyl-4,4'-diyl)di(1Z)hydrazin-2-yl-1ylidene]bis(5 -amino-4-oxo-3,4-dihydronaphthalene-2,7-disulfonic acid) as shown in Figure 7.

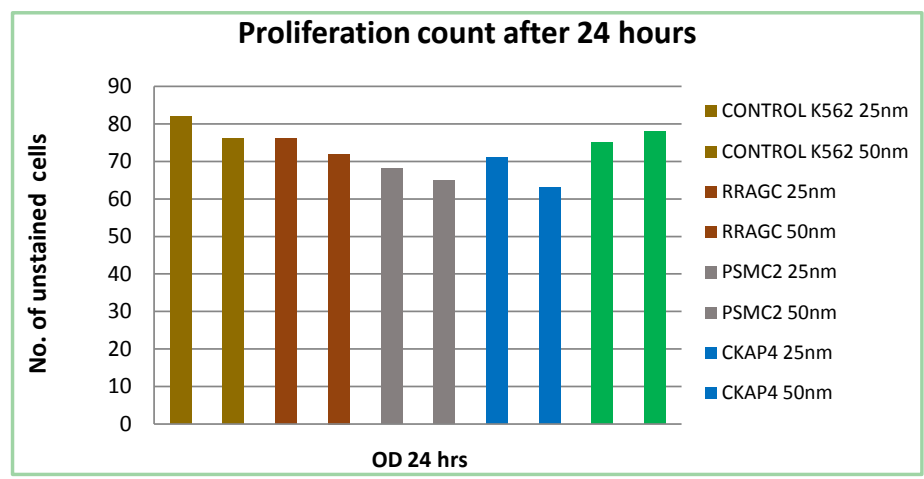

(a) Proliferation count after 24 hours

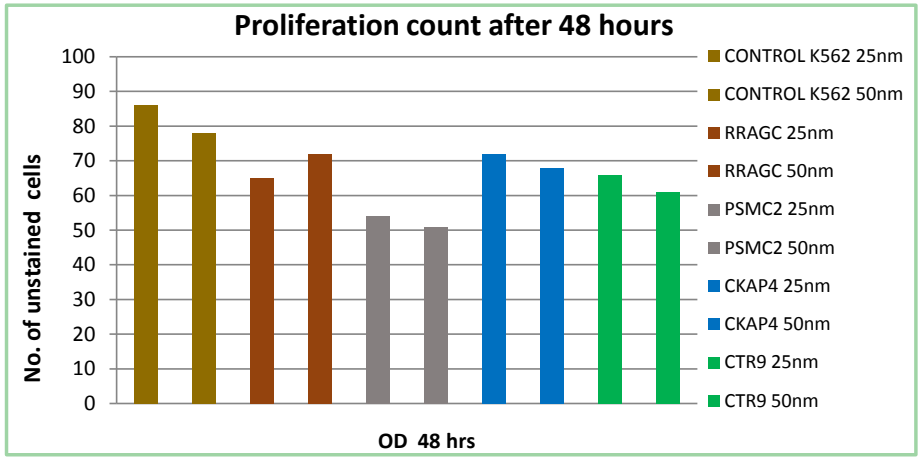

(b) Proliferation count after 48 hours

Figure 7. Proliferation count after siRNA knock down in (a) 24 hours and (b) 48 hours. 


\section{Conclusions}

Several potential underlying aspects of interaction among the gene pairs viz. RRAGC \& PSMC2; CKAP4 \& MANF and CTR9 \& CNTNAP2 have been studied in the context of their roles in proteasomal degradation, ER/Golgi Signaling, and dopamine mediated signaling respectively. The synergistic effect of RRAGC \& PSMC2 governing the proteasomal degradation mainly through ubiquitination has been shown to stimulate autophagy to remove toxic proteins. While, the interaction of CKAP4 and MANF is shown apparently through binding ATF6 and through KDEL, that are engaged in ER/Golgi Signaling. Further, CTR9 and CNTNAP2 have a profound effect in dopamine-mediated signaling through the ion-gradient and C-terminal binding site for a PDZ domain.

However, much remains obscure about their synergistic effects in signaling pathways, mechanical cues, and their role in the regulation of haematopoiesis. However, novel strategies like the expression of these genes in stem cell line together with targeted siRNA gene knock down procedures and functional analysis by measuring cell viability would provide important insights and reveal mechanisms governing the downstream cascades among interacting partners and their functional implications in signal transduction affecting hematopoietic stem cell proliferation and determine their therapeutic importance.

\section{Conflicts of Interest}

The authors declare no competing or financial interests.

\section{References}

[1] Kim, A., Stachura, D. and Traver, D. (2014) Cell Signaling Pathways Involved in Hematopoietic Stem Cell Specification. Experimental Cell Research, 329, 227-233. https://doi.org/10.1016/j.yexcr.2014.10.011

[2] Chotinantakul, K. and Leeanansaksiri, W. (2012) Hematopoietic Stem Cell Development, Niches, and Signaling Pathways. Bone Marrow Research, 2012, Article ID: 270425.

[3] Smithgall, T.E. (1998) Signal Transduction Pathways Regulating Hematopoietic Differentiation. Pharmacological Reviews, 50, 1-19.

[4] Guo, H.B., Isserlin, R., Chen, X.J., Wang, W.J., Phanse, S., Zandstra, P.W., Paddison, P.J. and Emili, A. (2013) Integrative Network Analysis of Signaling in Human CD34 ${ }^{+}$Hematopoietic Progenitor Cells by Global Phosphoproteomic Profiling Using $\mathrm{TiO}_{2}$ Enrichment Combined with 2D LC-MS/MS and Pathway Mapping. Proteomics, 13, 1325-1333. https://doi.org/10.1002/pmic.201200369

[5] Sharma, S., Gurudutta, G.U. and Singh, U. (2017) Stem Cell Genes Synergism: Elucidation of the Mechanistic Basis of Response Induced by Cytokine Combination (IL-3, FLT-3 and SCF). Journal of Stem Cells, 12, 143-160.

[6] Laplante, M. and Sabatini, D.M. (2012) mTOR Signaling in Growth Control and Disease. Cell, 149, 274-293. https://doi.org/10.1016/j.cell.2012.03.017

[7] Mendoza, M.C., Er, E.E. and Blenis, J. (2011) The Ras-ERK and PI3K-mTOR Pathways: Cross-Talk and Compensation. Trends in Biochemical Sciences, 36, 320-328. https://doi.org/10.1016/j.tibs.2011.03.006 
[8] Pópulo, H., Lopes, J.M. and Soares, P. (2012) The mTOR Signalling Pathway in Human Cancer. International Journal of Molecular Sciences, 13, 1886-1918. https://doi.org/10.3390/ijms13021886

[9] Sekiguchi, T., Hirose, E., Nakashima, N., Ii, M. and Nishimoto, T. (2001) Novel G Proteins, Rag C and Rag D, Interact with GTP-Binding Proteins, Rag A and Rag B. The Journal of Biological Chemistry, 276, 7246-7257.

https://doi.org/10.1074/jbc.M004389200

[10] Berridge, M.J. (2014) Cell Signaling Biology.

[11] Zhao, J.H.., Zhai, B., Gygi, S.P. and Goldberg, A.L. (2015) mTOR Inhibition Activates Overall Protein Degradation by the Ubiquitin Proteasome System as Well as by Autophagy. Proceedings of the National Academy of Sciences of the United States of America, 112, 15790-15797. https://doi.org/10.1073/pnas.1521919112

[12] Kanayama, H.O., Tamura, T., Ugai, S., Kagawa, S., Tanahashi, N., Yoshimura, T., Tanaka, K. and Ichihara, A. (1992) Demonstration That a Human 26S Proteolytic Complex Consists of a Proteasome and Multiple Associated Protein Components and Hydrolyzes ATP and Ubiquitin-Ligated Proteins by Closely Linked Mechanisms. European Journal of Biochemistry, 206, 567-578. https://doi.org/10.1111/j.1432-1033.1992.tb16961.x

[13] Tuffy, K.M. and Planey, S.L. (2012) Cytoskeleton-Associated Protein 4: Functions Beyond the Endoplasmic Reticulum in Physiology and Disease. International Scholarly Research Notices, 2012, Article ID: 142313.

[14] Nikonov, A.V., Hauri, H.P., Lauring, B. and Kreibich, G. (2007) Climp-63-Mediated Binding of Microtubules to the ER Affects the Lateral Mobility of Translocon Complexes. Journal of Cell Science, 120, 2248-2258. https://doi.org/10.1242/jcs.008979

[15] Klopfenstein, D.R.S., Kappeler, F. and Hauri, H.P. (1998) A Novel Direct Interaction of Endoplasmic Reticulum with Microtubules. The EMBO Journal, 17, 6168-6177. https://doi.org/10.1093/emboj/17.21.6168

[16] Ortega, A., Roselló-Lletí, E., Tarazón, E., Molina-Navarro, M.M., Martínez-Dolz, L. and González-Juanatey, J.R. (2014) Endoplasmic Reticulum Stress Induces Different Molecular Structural Alterations in Human Dilated and Ischemic Cardiomyopathy. PLoS ONE, 9, e107635. https://doi.org/10.1371/journal.pone.0107635

[17] Lee, C. and Chen, L.B. (1988) Dynamic Behavior of Endoplasmic Reticulum in Living Cells. Cell, 54, 37-46. https://doi.org/10.1016/0092-8674(88)90177-8

[18] Schweizer, A., Peter, F., Van, P.N., Söling, H.D. and Hauri, H.P. (1993) A Luminal Calcium-Binding Protein with a KDEL Endoplasmic Reticulum Retention Motif in the ER-Golgi Intermediate Compartment. European Journal of Cell Biology, 60, 366-370.

[19] Kim, Y., Park, S.J. and Chen, Y.M. (2017) Mesencephalic Astrocyte-Derived Neurotrophic Factor (MANF), a New Player in Endoplasmic Reticulum Diseases: Structure, Biology, and Therapeutic Roles. Translational Research, 188, 1-9. https://doi.org/10.1016/j.trsl.2017.06.010

[20] Henderson, M.J., Richie, C.T., Airavaara, M., Wang, Y. and Harvey, B.K. (2013) Mesencephalic Astrocyte-Derived Neurotrophic Factor (MANF) Secretion and Cell Surface Binding Are Modulated by KDEL Receptors. The Journal of Biological Chemistry, 288, 4209-4225. https://doi.org/10.1074/jbc.M112.400648

[21] De Young, M.B., Keller, J.C., Graham, R.M. and Wildey, G.M. (1994) Brefeldin A Defines Distinct Pathways for Atrial Natriuretic Factor Secretion in Neonatal rat Atrial and Ventricular Myocytes. Circulation Research, 74, 33-40. 
https://doi.org/10.1161/01.RES.74.1.33

[22] Petrova, P., Raibekas, A., Pevsner, J. Vigo, N., Anafi, M., Moore, M.K., et al. (2003) MANF. A New Mesencephalic, Astrocyte-Derived Neurotrophic Factor with Selectivity for Dopaminergic Neurons. Journal of Molecular Neuroscience, 20, 173-188. https://doi.org/10.1385/JMN:20:2:173

[23] Montero, M., Barrero, M.J. and Alvarez, J. (1997) $\mathrm{Ca}^{2+}$ Microdomains Control Agonist-Induced $\mathrm{Ca}^{2+}$ Release in Intact HeLa Cells. The FASEB Journal, 11, 881-885. https://doi.org/10.1096/fasebj.11.11.9285486

[24] Meldolesi, J. and Pozzan, T. (1998) The Endoplasmic Reticulum Ca ${ }^{2+}$ Store: A View from the Lumen. Trends in Biochemical Sciences, 23, 10-14. https://doi.org/10.1016/S0968-0004(97)01143-2

[25] Treiman, M. (2002) Regulation of the Endoplasmic Reticulum Calcium Storage during the Unfolded Protein Response. Significance in Tissue Ischemia? Trends in Cardiovascular Medicine, 12, 57-62. https://doi.org/10.1016/S1050-1738(01)00147-5

[26] Glembotski, C.C., Thuerauf, D.J., Huang, C.Q., Vekich, J.A., Gottlieb, R.A. and Doroudgar, S. (2012) Mesencephalic Astrocyte-Derived Neurotrophic Factor Protects the Heart from Ischemic Damage and Is Selectively Secreted upon Sarco/Endoplasmic Reticulum Calcium Depletion. The Journal of Biological Chemistry, 287, 25893-5904. https://doi.org/10.1074/jbc.M112.356345

[27] Zeng, H. and Xu, W. (2015) Ctr9, a Key Subunit of PAFc, Affects Global Estrogen Signaling and Drives ER $\alpha$-Positive Breast Tumorigenesis. Genes \& Development, 29, 2153-2167. https://doi.org/10.1101/gad.268722.115

[28] Levin, E.R. (2005) Integration of the Extranuclear and Nuclear Actions of Estrogen. Molecular Endocrinology, 19, 1951-959. https://doi.org/10.1210/me.2004-0390

[29] De Gois, S., Slama, P., Pietrancosta, N., Erdozain, A.M., Louis, F., Bouvrais-Veret, C., Daviet, L. and Giros, B. (2015) Ctr9, a Protein in the Transcription Complex Paf1, Regulates Dopamine Transporter Activity at the Plasma Membrane. The Journal of Biological Chemistry, 290, 17848-17862. https://doi.org/10.1074/jbc.M115.646315

[30] Nieoullon, A. (2002) Dopamine and the Regulation of Cognition and Attention. Progress in Neurobiology, 67, 53-83.

https://doi.org/10.1016/S0301-0082(02)00011-4

[31] Giros, B. and Caron, M.G. (1993) Molecular Characterization of the Dopamine Transporter. Trends in Pharmacological Sciences, 14, 43-49. https://doi.org/10.1016/0165-6147(93)90029-J

[32] Chang, M.-Y., Lee, S.-H., Kim, J.-H., Lee, K.-H., Kim, Y.-S., Son, H. and Lee, Y.-S. (2001) Protein Kinase C-Mediated Functional Regulation of Dopamine Transporter Is Not Achieved by Direct Phosphorylation of the Dopamine Transporter Protein. Journal of Neurochemistry, 77, 754-761. https://doi.org/10.1046/j.1471-4159.2001.00284.x

[33] Lee, F.J.S., Liu, F., Pristupa, Z.B. and Niznik, H.B. (2001) Direct Binding and Functional Coupling of $\alpha$-Synuclein to the Dopamine Transporters Accelerate Dopamine-Induced Apoptosis. The FASEB Journal, 15, 916-926. https://doi.org/10.1096/fsb2fj000334com

[34] Cao, J., Dwyer, J.B., Mangold, J.E., Wang, J., Wei, J., Leslie, F.M., et al. (2011) Modulation of Cell Adhesion Systems by Prenatal Nicotine Exposure in Limbic Brain Regions of Adolescent Female Rats. International Journal of Neuropsychopharmacology, 14, 157-174. https://doi.org/10.1017/S1461145710000179

[35] Rzehak, P., Saffery, R., Reischl, E., Covic, M., Wahl, S., Grote, V., et al. (2016) Ma- 
ternal Smoking during Pregnancy and DNA-Methylation in Children at Age 5.5 Years: Epigenome-Wide-Analysis in the European Childhood Obesity Project (CHOP)-Study. PLoS ONE, 11, e0155554.

https://doi.org/10.1371/journal.pone.0155554

[36] Denisenko-Nehrbass, N., Oguievetskaia, K., Goutebroze, L., Galvez, T., Yamakawa, H., Ohara, O., Carnaud, M. and Girault, J.-A. (2003) Protein 4.1B Associates with Both Caspr/Paranodin and Caspr2 at Paranodes and Juxtaparanodes of Myelinated Fibres. European Journal of Neuroscience, 17, 411-416.

https://doi.org/10.1046/j.1460-9568.2003.02441.x

[37] Hemming, N.J., Anstee, D.J., Mawby, W.J., Reid, M.E. and Tanner, M.J. (1994) Localization of the Protein 4.1-Bindimng Site on Human Erythrocyte Glycophorins C and D. Biochemical Journal, 299, 191-196.https://doi.org/10.1042/bj2990191

[38] Marfatia, S.M., Lue, R.A., Branton, D. and Chishti, A.H. (1995) Identification of the Protein 4.1 Binding Interface on Glycophorin C and p55, a Homologue of the Drosophila Discs-Large Tumor Suppressor Protein. The Journal of Biological Chemistry, 270, 715-719. https://doi.org/10.1074/jbc.270.2.715

[39] Dupree, J.L., Girault, J.A. and Popko, B. (1999) Axo-Glial Interactions Regulate the Localization of Axonal Paranodal Proteins. Journal of Cell Biology, 147, 1145-1152. https://doi.org/10.1083/jcb.147.6.1145

[40] Peles, E., Nativ, M., Lustig, M., Grumet, M., Schilling, J., Martinez, R., et al. (1996) Identification of a Novel Contac-Tin-Associated Transmembrane Receptor with Multiple Domains Implicated in Protein-Protein Interactions. The EMBO Journal, 16, 978-988. https://doi.org/10.1093/emboj/16.5.978 